\title{
Combining MicroED and GIWAXS for determining structure and orientation of organic semiconductor thin films
}

\author{
Brent Nannenga \\ Arizona State University, Tempe, United States of America; \\ brent.nannenga@asu.edu
}

In order to understand the packing and orientation of organic semiconductor thin films, we make use of microcrystal electron diffraction (MicroED) and grazing-incidence wide-angle X-ray scattering (GIWAXS). These complementary techniques provide structural insights to the structure of these thin films and can be used with the same sample preparation methods that are used to create the functional films. This removes the need for time-consuming crystallization experiments that may not directly capture the same semiconductor structure found in the films. We will present the application of these methods on four organic semiconductor samples, some of which represent novel structures determined by MicroED.

Keywords: MicroED, organic semiconductors, electron diffraction, GIWAXS 